\title{
Decomposition Solution for Nonlinear Model Describing Diffusional Growth of Intermetallic Layers
}

\author{
H. FATOOREHCHI ${ }^{a, *}$ AND R. RACH ${ }^{b}$ \\ ${ }^{a}$ School of Chemical Engineering, College of Engineering, \\ University of Tehran, P.O. Box 11155-4563, Tehran, Iran \\ ${ }^{b}$ The George Adomian Center for Applied Mathematics, \\ 316 South Maple Str., Hartford, MI 49057-1225, USA \\ Received: 30.03.2021 \& Accepted: 05.05.2021 \\ Doi: 10.12693/APhysPolA.140.91 \\ *e-mail: hfatoorehchi@ut.ac.ir

\begin{abstract}
We investigate the solution of a system of two nonlinear ordinary differential equations that are aimed to represent the diffusional growth of intermetallic layers. Firstly, we formulate an equivalent secondorder ordinary differential equation for the general system. Afterwards, to treat the model analytically, we apply a powerful, yet simple method known as the Adomian decomposition method to calculate the convergent sequence of analytic functions which approximate the exact solution of the original problem as closely as we desire. For the sake of illustration, a real-world example is presented modeling the growth dynamics of $\mathrm{Al}_{3} \mathrm{Mg}_{2}$ and $\mathrm{Al}_{12} \mathrm{Mg}_{17}$. An excellent agreement is obtained between the experimental data and the model estimates.
\end{abstract}

topics: intermetallics, diffusion, kinetics, Adomian decomposition

\section{Introduction}

Intermetallic compounds (ICs) have attracted the attention of scientists worldwide for several decades because of their desirable physical, thermal, and mechanical properties. These materials constitute a novel class of structural compounds with certain characteristics superior over conventional alloys and ceramics. Particularly, the ICs exhibit low density, high mechanical strength, anti-corrosion properties, etc. [1]. For instance, intermetallic hydrides, as a specific class of the ICs, tend to adsorb hydrogen gas and hence they are being studied as promising candidates for hydrogen storage applications [2]. Different methods for the synthesis of ICs have been used extensively. They include powder metallurgy, self-combustion synthesis, mechanical alloying, and electric current assisted sintering [3].

It is well known that intermetallic compounds form continuous layers at phase interfaces during a number of processes, such as in the hot-dip protective coating of solid surfaces with metals, soldering, welding of dissimilar metals or alloys, etc. [4-9]. To model the diffusional growth kinetics of intermetallic layers, parabolic functions are used, like $x^{2}=2 k t$, where $x, k$, and $t$ denote the layer thickness, layer growth kinetic constant, and time, respectively. Such a simple mathematical model closely matches the experimental data in the case of sufficiently thick layers $[10,11]$.

\section{Diffusional growth dynamics}

The diffusional growth dynamics of alloys $A_{p} B_{q}$ and $A_{r} B_{s}$ between simple species $A$ and $B$ appears to be much more complicated. In fact, it is more authentically modeled by a system of highly nonlinear ordinary differential equations with negative-power nonlinearities [12]:

$$
\begin{aligned}
& \frac{\mathrm{d} x}{\mathrm{~d} t}=\frac{k_{A}}{x}-\frac{r g}{p} \frac{k_{B}}{y}, \\
& \frac{\mathrm{d} y}{\mathrm{~d} t}=\frac{k_{B}}{y}-\frac{q}{s g} \frac{k_{A}}{x},
\end{aligned}
$$

where $x, y, k_{A}$ and $k_{B}$ denote, respectively, the layer thicknesses and growth rate constants for $A_{p} B_{q}$ and $A_{r} B_{s}$. Also, $g$ is the ratio of the molar volumes of the compounds $A_{p} B_{q}$ and $A_{r} B_{s}$.

The derivation of (1) and (2) can be briefly summarized as follows. As depicted in Fig. 1, the $A_{p} B_{q}$ layer thickness increases by the value $\mathrm{d} x_{D}$ due to the diffusion of $A$ atoms during the infinitesimal time increment $\mathrm{d} t$. Since the $A$ atoms have to diffuse through the layer thickness $x$, the value of $\mathrm{d} x_{D}$ is inversely proportional to $x$ and directly proportional to $\mathrm{d} t$. Thus, we can formulate this as

$$
\mathrm{d} x_{D}=\frac{k_{A}}{x} \mathrm{~d} t \text {. }
$$

However, one should realize that a partial chemical reaction takes place at interface 2 . Namely,

$$
(s p-q r) B_{\mathrm{diff}}+r A_{p} B_{q} \Rightarrow p A_{r} B_{s},
$$




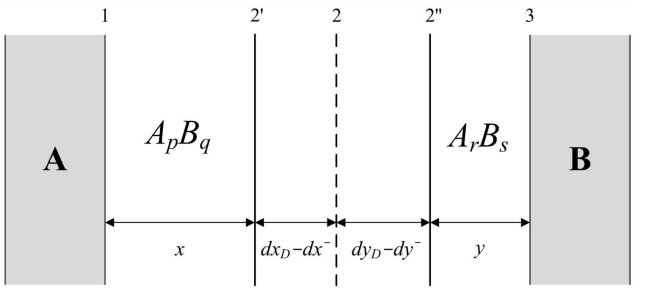

Fig. 1. Schematic illustration of the growth process of two intermetallic compound layers for the diffusion control case.

which means that the diffused $B$ atoms reach interface 2 and react with the $A_{p} B_{q}$ compound, and then form $A_{r} B_{s}$. In other words, the layer thickness of $A_{p} B_{q}$ is decreased by $\mathrm{d} x^{-}$. The net thickness change of $x$ becomes then

$$
\mathrm{d} x=\mathrm{d} x_{D}-\mathrm{d} x^{-} .
$$

Now, let us assume that $m_{A_{p} B_{q}}$ unit of mass of $A_{p} B_{q}$ participates in reaction (4) to produce $m_{A_{r} B_{s}}$ unit mass of $A_{r} B_{s}$. According to the stoichiometry, it follows that:

$$
\frac{m_{A_{p} B_{q}}}{m_{A_{r} B_{s}}}=\frac{r M_{A_{p} B_{q}}}{p M_{A_{r} B_{s}}},
$$

which leads to

$$
\frac{\rho_{A_{p} B_{q}} \mathrm{~d} x^{-}}{\rho_{A_{r} B_{s}} \mathrm{~d} y_{D}}=\frac{r}{p} \frac{M_{A_{p} B_{q}}}{M_{A_{r} B_{s}}},
$$

and finally one has

$$
\mathrm{d} x^{-}=\frac{r g}{p} \mathrm{~d} y_{D} \text {. }
$$

Now, we can substitute (8) and (3) into (5), and obtain

$$
\mathrm{d} x=\frac{k_{A}}{x} \mathrm{~d} t-\frac{r g}{p} \mathrm{~d} y_{D} .
$$

Similarly to (3), for atoms $B$, we can have

$$
\mathrm{d} y_{D}=\frac{k_{B}}{y} \mathrm{~d} t \text {. }
$$

From (9) and (10), we can obtain the nonlinear ordinary differential equation (ODE) (1). In a similar manner, (2) can also be derived.

This paper aims to investigate the diffusional growth of intermetallic layers through this complicated model by means of a powerful analytic method known as the Adomian decomposition method. Since no simplifying assumption is made to (1) and (2) in the course of our solution, the obtained results are extremely accurate. For illustration, we analyze a real-world case with the study of the growth dynamics of $\mathrm{Al}_{3} \mathrm{Mg}_{2}$ and $\mathrm{Al}_{12} \mathrm{Mg}_{17}$ in the sequel.

\section{Fundamentals of Adomian decomposition method}

A quick review of the basics of the ADM is presented in this section.

The Adomian decomposition method (ADM) is able to treat a wide class of nonlinear functional equations including differential, integral, integrodifferential, algebraic, etc. [13-15]. There is a considerable literature on applications of the ADM to various problems in science and engineering, e.g., see [16-28].

Without loss of generality, let us consider the following nonlinear differential equation:

$$
L u+N u+R u=g,
$$

where $L$ denotes the easily invertible linear operator, $N$ is the nonlinear operator, $R u$ designates the remaining parts of the equation under consideration, and $g$ is the specified bounded function. In our case, $L$ is the highest-order linear differential operator. Now, one could apply the inverse operator $L^{-1}$, i.e., the chosen integral operator, to both sides of (11). Then, one obtains

$$
u=a-L^{-1} g-L^{-1} N u-L^{-1} R u,
$$

where $a$ is the parameter resulting from the integrations such that $L a=0$. For the case of a first-order ODE, $a$ is the constant of integration.

The ADM assumes that the solution to (11) is in the form of an infinite series as $u=\sum_{n=0}^{+\infty} u_{n}$. Here, the solution components $u_{n}$ are to be determined by recursion. The ADM also assumes a decomposition of the nonlinear terms as $N u=\sum_{n=0}^{+\infty} A_{n}$, where the $A_{n}$ are the Adomian polynomials. By definition, the $A_{n}$ can be calculated as

$$
\begin{aligned}
& A_{n}\left(u_{0}, u_{1}, \ldots, u_{n}\right)= \\
& \quad \frac{1}{n !}\left[\frac{\mathrm{d}^{n}}{\mathrm{~d} \lambda^{n}} N\left(\sum_{i=0}^{+\infty} \lambda^{i} u_{i}\right)\right]_{\lambda=0} .
\end{aligned}
$$

With the use of (12) and the appropriate decompositions assumed by the ADM, as well as with the choice of the initial solution component to be $u_{0}=a-L^{-1} g$, one can construct the recursion scheme to obtain all the solution components. This is given as follows:

$$
\left\{\begin{array}{l}
u_{0}=a-L^{-1} g \\
u_{i+1}=-L^{-1} A_{i}-L^{-1} R u_{i}, \quad i \geq 0 .
\end{array}\right.
$$

The convergence of the ADM has been proved in [29-32]. Other efficient techniques for calculating the Adomian polynomials are available in the literature [33-37].

\section{Solution of the model by ADM}

Although the ADM can be readily applied to solve systems of ODEs [38], however, it might be more convenient to combine (1) and (2) into an equivalent second-order ODE and thus convert our problem to a single ODE. To do so, we first search for the solution for the function $x$ from (2). It is in fact

$$
x=\frac{q k_{A}}{s g}\left(\frac{k_{B}}{y}-\frac{\mathrm{d} y}{\mathrm{~d} t}\right)^{-1} .
$$


Based on (15), we next calculate the derivative of $x$ with respect to $t$, i.e.

$$
\frac{\mathrm{d} x}{\mathrm{~d} t}=\frac{q k_{A}}{s g}\left(\frac{k_{B}}{y}-\frac{\mathrm{d} y}{\mathrm{~d} t}\right)^{-2}\left(\frac{k_{B}}{y^{2}} \frac{\mathrm{d} y}{\mathrm{~d} t}+\frac{\mathrm{d}^{2} y}{\mathrm{~d} t^{2}}\right) .
$$

Substituting (15) and (16) into (1) and performing suitable algebraic rearrangements, one yields

$$
\begin{gathered}
\frac{\mathrm{d}^{2} y}{\mathrm{~d} t^{2}}=-\frac{k_{B}}{y^{2}} \frac{\mathrm{d} y}{\mathrm{~d} t}+\frac{s^{2} g^{2}}{q^{2} k_{A}}\left(\frac{k_{B}}{y}-\frac{\mathrm{d} y}{\mathrm{~d} t}\right)^{3} \\
-\frac{s g^{2} r k_{B}}{q p k_{A}} \frac{1}{y}\left(\frac{k_{B}}{y}-\frac{\mathrm{d} y}{\mathrm{~d} t}\right)^{2} .
\end{gathered}
$$

It turns out that (17) can be solved by the ADM when selecting the operator form as $L(\cdot)=\frac{\mathrm{d}^{2}}{\mathrm{~d} t^{2}}(\cdot)$. This also implies the following property of the inverse $L^{-1}(\cdot)=\int_{0}^{t} \mathrm{~d} t \int_{0}^{t} \mathrm{~d} t^{\prime}(\cdot)$.

Hence, according to the ADM, the solution of $(17)$ is

$$
y=\sum_{i=0}^{+\infty} y_{i},
$$

where the solution components are calculated as

$$
\left\{\begin{array}{l}
y_{0}=\alpha+\beta t \\
y_{i+1}=-k_{B} \int_{0}^{t} \mathrm{~d} t \int_{0}^{t} \mathrm{~d} t^{\prime} A_{i}+\frac{s^{2} g^{2}}{q^{2} k_{A}} \int_{0}^{t} \mathrm{~d} t \int_{0}^{t} \mathrm{~d} t^{\prime} B_{i} \\
\quad-\frac{s g^{2} r}{q p} \frac{k_{B}}{k_{A}} \int_{0}^{t} \mathrm{~d} t \int_{0}^{t} \mathrm{~d} t^{\prime} C_{i},
\end{array}\right.
$$

for $i \geq 0$. Two parameters, $\alpha$ and $\beta$, have to be determined by the initial conditions of the problem, i.e.

$$
\begin{aligned}
& \alpha=y(0), \\
& \beta=\frac{\mathrm{d}}{\mathrm{d} t} y(0) .
\end{aligned}
$$

In turn, $A_{i}, B_{i}$ and $C_{i}$ are the Adomian polynomials such that

$$
\begin{aligned}
& \sum_{i=0}^{+\infty} A_{i}=\frac{1}{y^{2}} \frac{\mathrm{d} y}{\mathrm{~d} t} \\
& \sum_{i=0}^{+\infty} B_{i}=\left(\frac{k_{B}}{y}-\frac{\mathrm{d} y}{\mathrm{~d} t}\right)^{3}, \\
& \sum_{i=0}^{+\infty} C_{i}=\left(\frac{k_{B}}{y}-\frac{\mathrm{d} y}{\mathrm{~d} t}\right)^{2} .
\end{aligned}
$$

We have listed the first components of the foregoing Adomian polynomials in Appendix.

Once the function $y$ has been evaluated, it is straightforward to calculate the function $x$ by virtue of (15).

\section{Application}

In this section, the above-mentioned solution strategy is applied to the description of the growth dynamics of $\mathrm{Al}_{3} \mathrm{Mg}_{2}$ and $\mathrm{Al}_{12} \mathrm{Mg}_{17}$ layers, denoted by $x$ and $y$, respectively. Our results are next compared with the experimental data from [39]. For this
TABLE I

Numerical values of the parameters which appear in (17), where $k_{A}$ and $k_{B}$ are the growth rate constant for $\mathrm{Al}_{3} \mathrm{Mg}_{2}$ and $\mathrm{Al}_{12} \mathrm{Mg}_{17}$ respectively; $g$ is the ratio of molar volumes of $\mathrm{Al}_{3} \mathrm{Mg}_{2}$ and $\mathrm{Al}_{12} \mathrm{Mg}_{17} ; \alpha$ is the initial layer thickness $y$; $\beta$ is the initial slope of $y$; $p, q, r, s$ are the stoichiometric coefficients.

\begin{tabular}{l|c}
\hline \hline Parameter & Value \\
\hline$k_{A}\left[\mathrm{~m}^{2} / \mathrm{s}\right]$ & $3.40 \times 10^{-13} \mathrm{~m}^{2} / \mathrm{s}$ \\
$k_{B}\left[\mathrm{~m}^{2} / \mathrm{s}\right]$ & $9.37 \times 10^{-14} \mathrm{~m}^{2} / \mathrm{s}$ \\
$g$ & 0.94 \\
$\alpha[\mathrm{m}]$ & $0.144 \times 10^{-14} \mathrm{~m}$ \\
$\beta[\mathrm{m} / \mathrm{s}]$ & $6 \times 10^{-10} \mathrm{~m} / \mathrm{s}$ \\
$p$ & 3 \\
$q$ & 2 \\
$r$ & 12 \\
$s$ & 17
\end{tabular}

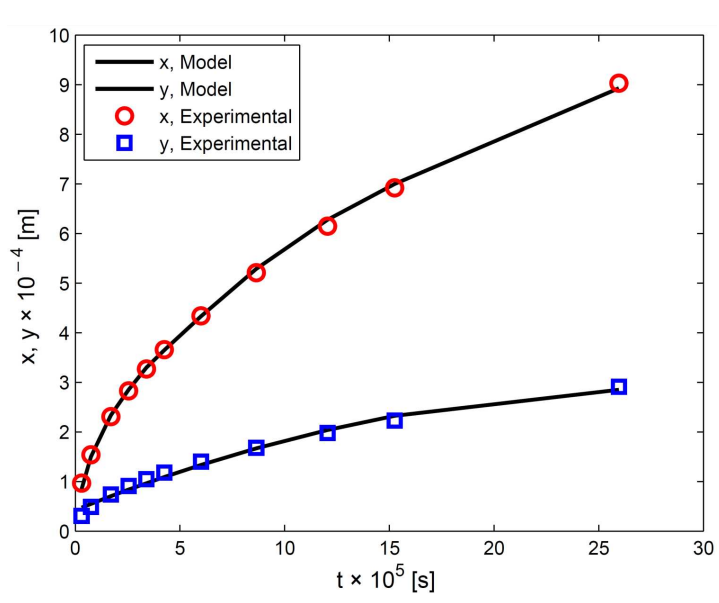

Fig. 2. Comparison of the experimental data and model estimates for layer thickness against annealing time for $\mathrm{Al}-\mathrm{Mg}$ diffusion couples at $400{ }^{\circ} \mathrm{C}$. The experimental data is due to Tanguep Nijokep et al. [39].

certain study, the numeric values for the invariants in (17) are listed in Table I [8, 39, 40]. Figure 2 depicts the results obtained from (18) and (19), truncated after ten components, and the experimental data. The satisfactory fit to experimental data results from the accuracy of the ADM, as well as the completeness of the mathematical model. Note that no simplifying assumptions were imposed for the sake of mathematical tractability.

\section{Conclusion}

A complicated mathematical model comprising a system of two highly nonlinear differential equations, which simulates the diffusional growth of intermetallic layers, was investigated in this paper. Initially, the system was converted into an equivalent second-order nonlinear ODE. Subsequently, we applied the Adomian decomposition method in 
order to treat the model in its authentic form. As a result, an accurate simulation of the phenomenon was achieved at the extremely satisfactory level. This was numerically ascertained by a comparison between the experimental data ex- tracted from [39] for the formed layers of $\left(\mathrm{Al}_{3} \mathrm{Mg}_{2}\right.$, $\left.\mathrm{Al}_{12} \mathrm{Mg}_{17}\right)$ and the model estimates. Due to many valuable advantages of the ADM, it is recommended to scientists who conduct dynamics studies on phenomena encountered in intermetallics science.

\section{Appendix: First five components of Adomian polynomials used in (17)}

$$
\begin{aligned}
& A_{0}=\frac{\mathrm{d} y_{0}}{\mathrm{~d} t} \frac{1}{y_{0}^{2}} \\
& A_{1}=-2 \frac{\mathrm{d} y_{0}}{\mathrm{~d} t} \frac{y_{1}}{y_{0}^{3}}+\frac{\mathrm{d} y_{1}}{\mathrm{~d} t} \frac{1}{y_{0}^{2}} \\
& A_{2}=3 \frac{\mathrm{d} y_{0}}{\mathrm{~d} t} \frac{y_{1}^{2}}{y_{0}^{4}}-2 \frac{\mathrm{d} y_{1}}{\mathrm{~d} t} \frac{y_{1}}{y_{0}^{3}}+\frac{\mathrm{d} y_{2}}{\mathrm{~d} t} \frac{1}{y_{0}^{2}} \\
& A_{3}=-4 \frac{\mathrm{d} y_{0}}{\mathrm{~d} t} \frac{y_{1}^{3}}{y_{0}^{5}}+3 \frac{\mathrm{d} y_{1}}{\mathrm{~d} t} \frac{y_{1}^{2}}{y_{0}^{4}}+6 \frac{\mathrm{d} y_{0}}{\mathrm{~d} t} \frac{y_{1} y_{2}}{y_{0}^{4}}-2 \frac{\mathrm{d} y_{2}}{\mathrm{~d} t} \frac{y_{1}}{y_{0}^{3}}-2 \frac{\mathrm{d} y_{1}}{\mathrm{~d} t} \frac{y_{2}}{y_{0}^{3}}-2 \frac{\mathrm{d} y_{0}}{\mathrm{~d} t} \frac{y_{3}}{y_{0}^{3}}+\frac{\mathrm{d} y_{3}}{\mathrm{~d} t} \frac{1}{y_{0}^{2}} \\
& A_{4}=5 \frac{\mathrm{d} y_{0}}{\mathrm{~d} t} \frac{y_{1}^{4}}{y_{0}^{6}}-4 \frac{\mathrm{d} y_{1}}{\mathrm{~d} t} \frac{y_{1}^{3}}{y_{0}^{5}}-12 \frac{\mathrm{d} y_{0}}{\mathrm{~d} t} \frac{y_{1}^{2} y_{2}}{y_{0}^{5}}+3 \frac{\mathrm{d} y_{2}}{\mathrm{~d} t} \frac{y_{1}^{2}}{y_{0}^{4}}+6 \frac{\mathrm{d} y_{1}}{\mathrm{~d} t} \frac{y_{1} y_{2}}{y_{0}^{4}}+3 \frac{\mathrm{d} y_{0}}{\mathrm{~d} t} \frac{y_{2}^{2}}{y_{0}^{4}}+6 \frac{\mathrm{d} y_{0}}{\mathrm{~d} t} \frac{y_{1} y_{3}}{y_{0}^{4}}-2 \frac{\mathrm{d} y_{3}}{\mathrm{~d} t} \frac{y_{1}}{y_{0}^{3}} \\
& -2 \frac{\mathrm{d} y_{2}}{\mathrm{~d} t} \frac{y_{2}}{y_{0}^{3}}-2 \frac{\mathrm{d} y_{1}}{\mathrm{~d} t} \frac{y_{3}}{y_{0}^{3}}-2 \frac{\mathrm{d} y_{0}}{\mathrm{~d} t} \frac{y_{4}}{y_{0}^{3}}+\frac{\mathrm{d} y_{4}}{\mathrm{~d} t} \frac{1}{y_{0}^{2}} \\
& B_{0}=\left(\frac{k_{B}}{y_{0}}-\frac{\mathrm{d} y_{0}}{\mathrm{~d} t}\right)^{3} \\
& B_{1}=-3\left(\frac{k_{B}}{y_{0}}-\frac{\mathrm{d} y_{0}}{\mathrm{~d} t}\right)^{2}\left(\frac{k_{B} y_{1}}{y_{0}^{2}}+\frac{\mathrm{d} y_{1}}{\mathrm{~d} t}\right) \\
& B_{2}=3\left(\frac{k_{B}}{y_{0}}-\frac{\mathrm{d} y_{0}}{\mathrm{~d} t}\right)\left(\frac{k_{B} y_{1}}{y_{0}^{2}}+\frac{\mathrm{d} y_{1}}{\mathrm{~d} t}\right)^{2} \\
& +3\left(\frac{k_{B}}{y_{0}}-\frac{\mathrm{d} y_{0}}{\mathrm{~d} t}\right)^{2}\left(\frac{k_{B} y_{1}^{2}}{y_{0}^{3}}-\frac{k_{B} y_{2}}{y_{0}^{2}}-\frac{\mathrm{d} y_{2}}{\mathrm{~d} t}\right) \\
& B_{3}=-\left(\frac{k_{B} y_{1}}{y_{0}^{2}}+\frac{\mathrm{d} y_{1}}{\mathrm{~d} t}\right)^{3}-6\left(\frac{k_{B}}{y_{0}}-\frac{\mathrm{d} y_{0}}{\mathrm{~d} t}\right)\left(\frac{k_{B} y_{1}}{y_{0}^{2}}+\frac{\mathrm{d} y_{1}}{\mathrm{~d} t}\right)\left(\frac{k_{B} y_{1}^{2}}{y_{0}^{3}}-\frac{k_{B} y_{2}}{y_{0}^{2}}-\frac{\mathrm{d} y_{2}}{\mathrm{~d} t}\right) \\
& -3\left(\frac{k_{B}}{y_{0}}-\frac{\mathrm{d} y_{0}}{\mathrm{~d} t}\right)^{2}\left(\frac{k_{B} y_{1}^{3}}{y_{0}^{4}}-2 \frac{k_{B} y_{1} y_{2}}{y_{0}^{3}}+\frac{k_{B} y_{3}}{y_{0}^{2}}+\frac{\mathrm{d} y_{3}}{\mathrm{~d} t}\right) \\
& B_{4}=3\left(\frac{k_{B} y_{1}}{y_{0}^{2}}+\frac{\mathrm{d} y_{1}}{\mathrm{~d} t}\right)^{2}\left(\frac{k_{B} y_{1}^{2}}{y_{0}^{2}}-\frac{k_{B} y_{2}}{y_{0}^{2}}-\frac{\mathrm{d} y_{2}}{\mathrm{~d} t}\right)+3\left(\frac{k_{B}}{y_{0}}-\frac{\mathrm{d} y_{0}}{\mathrm{~d} t}\right)\left(\frac{k_{B} y_{1}^{2}}{y_{0}^{3}}-\frac{k_{B} y_{2}}{y_{0}^{2}}-\frac{\mathrm{d} y_{2}}{\mathrm{~d} t}\right)^{2} \\
& +6\left(\frac{k_{B}}{y_{0}}-\frac{\mathrm{d} y_{0}}{\mathrm{~d} t}\right)\left(\frac{k_{B} y_{1}}{y_{0}^{2}}+\frac{\mathrm{d} y_{1}}{\mathrm{~d} t}\right)\left(\frac{k_{B} y_{1}^{3}}{y_{0}^{4}}-2 \frac{k_{B} y_{1} y_{2}}{y_{0}^{3}}+\frac{k_{B} y_{3}}{y_{0}^{2}}+\frac{\mathrm{d} y_{3}}{\mathrm{~d} t}\right) \\
& +3\left(\frac{k_{B}}{y_{0}}-\frac{\mathrm{d} y_{0}}{\mathrm{~d} t}\right)^{2}\left(\frac{k_{B} y_{1}^{4}}{y_{0}^{5}}-3 \frac{k_{B} y_{1}^{2} y_{2}}{y_{0}^{4}}+\frac{k_{B} y_{2}^{2}}{y_{0}^{3}}+2 \frac{k_{B} y_{1} y_{3}}{y_{0}^{3}}-\frac{k_{B} y_{4}}{y_{0}^{2}}-\frac{\mathrm{d} y_{4}}{\mathrm{~d} t}\right) \\
& C_{0}=\frac{1}{y_{0}}\left(\frac{k_{B}}{y_{0}}-\frac{\mathrm{d} y_{0}}{\mathrm{~d} t}\right)^{2} \\
& C_{1}=-\frac{y_{1}}{y_{0}^{2}}\left(\frac{k_{B}}{y_{0}}-\frac{\mathrm{d} y_{0}}{\mathrm{~d} t}\right)^{2}-2\left(\frac{k_{B}}{y_{0}}-\frac{\mathrm{d} y_{0}}{\mathrm{~d} t}\right)\left(\frac{k_{B} y_{1}}{y_{0}^{2}}+\frac{\mathrm{d} y_{1}}{\mathrm{~d} t}\right) \frac{1}{y_{0}} \\
& C_{2}=\left(\frac{k_{B}}{y_{0}}-\frac{\mathrm{d} y_{0}}{\mathrm{~d} t}\right)^{2} \frac{y_{1}^{2}}{y_{0}^{3}}+2\left(\frac{k_{B}}{y_{0}}-\frac{\mathrm{d} y_{0}}{\mathrm{~d} t}\right)\left(\frac{k_{B} y_{1}}{y_{0}^{2}}+\frac{\mathrm{d} y_{1}}{\mathrm{~d} t}\right) \frac{y_{1}}{y_{0}^{2}}-\left(\frac{k_{B}}{y_{0}}-\frac{\mathrm{d} y_{0}}{\mathrm{~d} t}\right)^{2} \frac{y_{2}}{y_{0}^{2}} \\
& +\left(\frac{k_{B} y_{1}}{y_{0}^{2}}+\frac{\mathrm{d} y_{1}}{\mathrm{~d} t}\right)^{2} \frac{1}{y_{0}}+2\left(\frac{k_{B}}{y_{0}}-\frac{\mathrm{d} y_{0}}{\mathrm{~d} t}\right)\left(\frac{k_{B} y_{1}^{2}}{y_{0}^{3}}-\frac{k_{B} y_{2}}{y_{0}^{2}}-\frac{\mathrm{d} y_{2}}{\mathrm{~d} t}\right) \frac{1}{y_{0}}
\end{aligned}
$$




$$
\begin{aligned}
& C_{3}=-\left(\frac{k_{B}}{y_{0}}-\frac{\mathrm{d} y_{0}}{\mathrm{~d} t}\right)^{2} \frac{y_{1}^{3}}{y_{0}^{4}}-2\left(\frac{k_{B}}{y_{0}}-\frac{\mathrm{d} y_{0}}{\mathrm{~d} t}\right)\left(\frac{k_{B} y_{1}}{y_{0}^{2}}+\frac{\mathrm{d} y_{1}}{\mathrm{~d} t}\right) \frac{y_{1}^{2}}{y_{0}^{3}}+2\left(\frac{k_{B}}{y_{0}}-\frac{\mathrm{d} y_{0}}{\mathrm{~d} t}\right)^{2} \frac{y_{1} y_{2}}{y_{0}^{3}} \\
& -\left(\frac{k_{B} y_{1}}{y_{0}^{2}}+\frac{\mathrm{d} y_{1}}{\mathrm{~d} t}\right)^{2} \frac{y_{1}}{y_{0}^{2}}+2\left(\frac{k_{B}}{y_{0}}-\frac{\mathrm{d} y_{0}}{\mathrm{~d} t}\right)\left(\frac{k_{B} y_{1}}{y_{0}^{2}}+\frac{\mathrm{d} y_{1}}{\mathrm{~d} t}\right) \frac{y_{2}}{y_{0}^{2}}-\left(\frac{k_{B}}{y_{0}}-\frac{\mathrm{d} y_{0}}{\mathrm{~d} t}\right)^{2} \frac{y_{3}}{y_{0}^{2}} \\
& -2\left(\frac{k_{B} y_{1}}{y_{0}^{2}}+\frac{\mathrm{d} y_{1}}{\mathrm{~d} t}\right)\left(\frac{k_{B} y_{1}^{2}}{y_{0}^{3}}-\frac{k_{B} y_{2}}{y_{0}^{2}}-\frac{\mathrm{d} y_{2}}{\mathrm{~d} t}\right) \frac{1}{y_{0}} \\
& -2\left(\frac{k_{B}}{y_{0}}-\frac{\mathrm{d} y_{0}}{\mathrm{~d} t}\right)\left(\frac{k_{B} y_{1}^{3}}{y_{0}^{4}}-2 \frac{k_{B} y_{1} y_{2}}{y_{0}^{3}}+\frac{k_{B} y_{3}}{y_{0}^{2}}+\frac{\mathrm{d} y_{3}}{\mathrm{~d} t}\right) \frac{1}{y_{0}} \\
& C_{4}=2\left(\frac{k_{B}}{y_{0}}-\frac{\mathrm{d} y_{0}}{\mathrm{~d} t}\right)\left(\frac{k_{B} y_{1}^{4}}{y_{0}^{5}}-3 \frac{k_{B} y_{1}^{2} y_{2}}{y_{0}^{4}}+\frac{k_{B} y_{2}^{2}}{y_{0}^{3}}+2 \frac{k_{B} y_{1} y_{3}}{y_{0}^{3}}-\frac{k_{B} y_{4}}{y_{0}^{2}}-\frac{\mathrm{d} y_{4}}{\mathrm{~d} t}\right) \frac{1}{y_{0}} \\
& +2\left(\frac{k_{B} y_{1}}{y_{0}^{2}}+\frac{\mathrm{d} y_{1}}{\mathrm{~d} t}\right)\left(\frac{k_{B} y_{1}^{3}}{y_{0}^{4}}-2 \frac{k_{B} y_{1} y_{2}}{y_{0}^{3}}+\frac{k_{B} y_{3}}{y_{0}^{2}}+\frac{\mathrm{d} y_{3}}{\mathrm{~d} t}\right) \frac{1}{y_{0}}-\left(\frac{k_{B} y_{1}}{y_{0}^{2}}+\frac{\mathrm{d} y_{1}}{\mathrm{~d} t}\right)^{2} \frac{y_{2}}{y_{0}^{2}} \\
& +\left(\frac{k_{B} y_{1}^{2}}{y_{0}^{3}}-\frac{k_{B} y_{2}}{y_{0}^{2}}-\frac{\mathrm{d} y_{2}}{\mathrm{~d} t}\right)^{2} \frac{1}{y_{0}}-\left(\frac{k_{B}}{y_{0}}-\frac{\mathrm{d} y_{0}}{\mathrm{~d} t}\right)^{2} \frac{y_{4}}{y_{0}^{2}}+\left(\frac{k_{B}}{y_{0}}-\frac{\mathrm{d} y_{0}}{\mathrm{~d} t}\right)^{2} \frac{y_{1}^{4}}{y_{0}^{5}} \\
& +2\left(\frac{k_{B}}{y_{0}}-\frac{\mathrm{d} y_{0}}{\mathrm{~d} t}\right)\left(\frac{k_{B} y_{1}}{y_{0}^{2}}+\frac{\mathrm{d} y_{1}}{\mathrm{~d} t}\right) \frac{y_{1}^{3}}{y_{0}^{4}}-3\left(\frac{k_{B}}{y_{0}}-\frac{\mathrm{d} y_{0}}{\mathrm{~d} t}\right)^{2} \frac{y_{1}^{2} y_{2}}{y_{0}^{4}} \\
& +\left(\frac{k_{B} y_{1}}{y_{0}^{2}}+\frac{\mathrm{d} y_{1}}{\mathrm{~d} t}\right)^{2} \frac{y_{1}^{2}}{y_{0}^{3}}+2\left(\frac{k_{B}}{y_{0}}-\frac{\mathrm{d} y_{0}}{\mathrm{~d} t}\right)\left(\frac{k_{B} y_{1}^{2}}{y_{0}^{3}}-\frac{k_{B} y_{2}}{y_{0}^{2}}-\frac{\mathrm{d} y_{2}}{\mathrm{~d} t}\right) \frac{y_{1}^{2}}{y_{0}^{3}}
\end{aligned}
$$

\section{Acknowledgments}

We are grateful to the Editor-in-Chief and anonymous reviewers of Acta Physica Polonica A for their insightful comments on the earlier draft of this paper.

\section{References}

[1] I. Prochazka, T. Vlasak, J. Cizek, F. Lukac, M.O. Liedke, W. Anwand, Y. Jiraskova, D. Janickovic, Acta Phys. Pol. A 137, 255 (2020).

[2] P. Bhardwaj, R. Bhardwaj, A.P. Mishra, Acta Phys. Pol. A 137, 1193 (2020).

[3] N. Ergin, O. Ozdemir, Acta Phys. Pol. A 125, 399 (2014).

[4] A.G. Metcalfe, Composite Materials, Academic Press, New York 1974.

[5] R.K. Everett, R.J. Arsenault, Metal Matrix Composites: Processing and Interfaces, Academic Press, New York 1991.

[6] G. Zhang, M. Chen, Y. Shi, J. Huang, F. Yang, RSC Adv. 7, 37797 (2017).

[7] Y. Wang, Y. Yao, L. Keer, J. Theor. Appl. Mech. Lett. 10, 33 (2020).

[8] O.V. Dybkov, V.I. Dybkov, J. Mater. Sci. 39, 6615 (2004).

[9] M.Z. Khalid, J. Friis, P.H. Ninive, K. Marthinsen, A. Strandlie, Comput. Mater. Sci. 174, 109481 (2020).

[10] W. Seith, Diffusion in Metallen, Springer, Berlin 1955.
[11] H. Mehrer, Diffusion in Solid Metals and Alloys, Springer, New York 1990.

[12] V.I. Dybkov, Reaction Diffusion and Solid State Chemical Kinetics, The IPMS Publications, Kiev 2002.

[13] G. Adomian, J. Math. Anal. Appl. 102, 420 (1984).

[14] G. Adomian, Math. Comput. Model. 13, 17 (1990).

[15] G. Adomian, Solving Frontier Problems of Physics: The Decomposition Method, Kluwer Academic, Dordrecht 1994.

[16] A. Alshaery, A. Ebaid, Acta Astronaut. 140, 27 (2017).

[17] M. Asma, W.A.M. Othman, B.R. Wong, A. Biswas, Optik 164, 632 (2018).

[18] J. Gambo, D. Gambo, Heat Transfer 50, 2288 (2021).

[19] S.A.S. Hashemi, H. Saeedi, Math. Comput. Simul. 185, 783 (2021).

[20] G.Y. Kim, C.H. Kwak, J. Opt. 22, 095502 (2020).

[21] A.-M. Wazwaz, R. Rach, J.-S. Duan, Appl. Math. Comput. 219, 5004 (2013).

[22] A. Barari, M. Rahimi, M. Hosseini, L. Ibsen, Acta Phys. Pol. A 122, 96 (2012).

[23] A.M.A. El-Sayed, M. Gaber, Phys. Lett. A 359, 175 (2006).

[24] H. Fatoorehchi, H. Abolghasemi, Intermetallics 32, 35 (2013). 
[25] H. Fatoorehchi, R. Rach, Alex. Eng. J. 59, 4879 (2020).

[26] H. Fatoorehchi, R. Rach, O. Tavakoli, H. Abolghasemi, Chem. Eng. Commun. 202, 402 (2015).

[27] D. Zeidan, C.K. Chau, T.-T. Lu, W.-Q. Zheng, Math. Meth. Appl. Sci. 43, 2171 (2020).

[28] J.S. Duan, R. Rach, D. Baleanu, A.-M. Wazwaz, Commun. Fract. Calc. 3, 73 (2012).

[29] K. Abbaoui, Y. Cherruault, Comput. Math. Appl. 28, 103 (1994).

[30] Y. Cherruault, G. Adomian, Math. Comput. Model. 18, 103 (1993).

[31] K. Abbaoui, Y. Cherruault, Math. Comput. Model. 20, 69 (1994).

[32] A. Abdelrazec, D. Pelinovsky, Numer. Meth. Part. Differ. Equat. 27, 749 (2011).
[33] G. Adomian, R. Rach, J. Math. Anal. Appl. 91, 39 (1983).

[34] R. Rach, Kybernetes 37, 910 (2008).

[35] J.S. Duan, Appl. Math. Comput. 217, 2456 (2010).

[36] J.S. Duan, Appl. Math. Comput. 216, 1235 (2010).

[37] J.S. Duan, Appl. Math. Comput. 217, 6337 (2011).

[38] J. Biazar, E. Babolian, R. Islam, Appl. Math. Comput. 147, 713 (2004).

[39] E.M. Tanguep Nijokep, M. Salamon, H. Mehrer, Def. Diff. Forum 194-199, 1581 (2001.

[40] L.F. Mondolfo, Aluminium Alloys: Structure and Properties, Butterworths, London 1976. 